\title{
STERNAL RESECTION FOR PRIMARY OR SECONDARY TUMORS
}

$\mathrm{M}$. Incarbone, $\mathrm{MD}^{\mathrm{a}}$

M. Nava, MD ${ }^{\mathrm{b}}$

C. Lequaglie, $\mathrm{MD}^{\mathrm{a}}$

G. Ravasi, MD

U. Pastorino, $\mathrm{MD}^{\mathrm{a}}$
Methods: From January 1980 to December 1993, 52 patients underwent surgical resection for tumors involving the sternum. The series included 20 primary malignant tumors, 4 desmoid tumors, 2 malignant tumors infiltrating the sternum from adjacent organs, 19 local recurrences or metastases of breast tumors, and 7 metastases of other tumors. Total sternectomy was performed in 5 patients, subtotal sternal resection in 19, and partial resection (less than $50 \%$ of the sternum) in 28 . Concurrent en bloc resection included anterior ribs in 37 patients, clavicle in 11, lung in 12 patients, pericardium in 7 , and diaphragm in 2 . The chest was reconstructed with prosthetic material and a myocutaneous flap in 26 patients $(50 \%)$, prosthetic material only in 12 patients (23\%), a myocutaneous flap in 5 patients $(10 \%)$, and other techniques in the remaining patients. In 47 patients $(90 \%)$ the resection was radical, and in the remaining 5 patients it was palliative. Results: No perioperative deaths occurred. After a median follow-up of 39 months, the overall 3-year survival was $58 \%$ and the 5 -year survival $46 \%$, with a median survival of 50 months. In 24 patients with primary tumor the 5 -year survival after radical resection was $63 \%$, and in 23 patients with secondary invasion (direct extension or metastasis) the 5-year survival was 38\% (median 35 months). In recurrent breast cancer the 5-year survival was $48 \%$ in patients with direct extension to the chest wall and $60 \%$ in patients with distant bone metastasis. Conclusions: Our experience demonstrates that sternal resection is a safe and effective treatment, which may improve the patient's quality of life and achieve a long-term survival not only in primary tumors but also in selected secondary malignant tumors of the sternum. (J Thorac Cardiovasc Surg 1997;114:93-9)
$T_{\mathrm{cos}}^{\mathrm{u}}$ umors of the sternum have been considered a challenging problem for a long time. Stability and reconstruction of chest wall defects have caused the main difficulties in radical full-thickness resections. Improvement of surgical techniques, especially by means of myocutaneous flaps and prosthetic materials, has resulted in successful sternectomies and simultaneous reconstructions.

The development of safe techniques for chest wall reconstruction has expanded the role of surgery in this field. Extensive chest wall resections, including

From the Divisions of Thoracic Surgery ${ }^{\mathrm{a}}$ and Plastic Surgery, ${ }^{\mathrm{b}}$ Istituto Nazionale Tumori, Milan, Italy.

Received for publication May 8, 1996; revisions requested July 3, 1996; revisions received March 5, 1997; accepted for publication March 5, 1997.

Address for reprints: Matteo Incarbone, MD, Division of Thoracic Surgery, Istituto Clinico Humanitas, via Manzoni, 56, Rozzano, Milano 20089, Italy.

Copyright (C) 1997 by Mosby-Year Book, Inc.

$0022-5223 / 97 \$ 5.00+0 \quad \mathbf{1 2 / 1 / 8 1 5 8 7}$ total sternectomy, can now be performed with virtually no operative mortality. ${ }^{1}$ A methyl methacrylate composite prosthesis within two layers of polypropylene mesh (Marlex mesh, Bard Implants, Billerica, Mass.) offers the best mechanical strength and external profile after total or subtotal sternectomy.

Surgical treatment should be considered the best option in the majority of patients with primary malignant tumors involving the sternum, ${ }^{2-5}$ and it can achieve a definitive cure. In high-grade sarcomas, induction chemotherapy or postoperative chemotherapy/radiotherapy (or both) may be necessary to consolidate the results of surgical treatment. ${ }^{6}$ Wide surgical resection with adequate margins is no longer limited by the size of the chest wall defect, because concurrent reconstruction with prosthetic materials with or without myocutaneous flaps is a feasible treatment in most departments of thoracic surgery.

The role of surgical treatment is still the subject of 
Table I. Patients' features

\begin{tabular}{lrrrrr} 
& & & & \multicolumn{2}{c}{ Total } \\
\cline { 5 - 6 } & Primary & Breast & Metastasis $^{*}$ & No. & $\%$ \\
\hline Age (yr) & & & & & \\
$0-29$ & 3 & 0 & 2 & 5 & 9 \\
$30-59$ & 17 & 9 & 5 & 31 & 60 \\
$>60$ & 4 & 10 & 2 & 16 & 31 \\
Sex & & & & & \\
$\quad$ Male & 14 & 0 & 7 & 21 & 40 \\
Female & 10 & 19 & 2 & 31 & 60 \\
Total & 24 & 19 & 9 & 52 & 100 \\
\hline
\end{tabular}

${ }^{*}$ Including two cases of direct extension from adjacent tumors.

controversy for secondary malignant tumors of the sternum, and particularly for recurrence or metastasis of breast cancer. ${ }^{7}$ In recurrent breast cancer, surgery has usually been performed for palliation after failure of radiation, but recently an increase in survival has been reported after resection. ${ }^{8,9}$ On the other hand, sternal resection of metastases from other distant tumors is occasionally performed with a purely palliative intent.

This article reports our experience with sternal resections for primary or secondary tumors.

\section{Materials and methods}

Between January 1980 and December 1993, at the Division of Thoracic Surgery of the Istituto Nazionale Tumori, 52 consecutive patients underwent sternal resection for tumors involving the sternum, costochondral cartilages, anterior ribs, parasternal soft tissues, or a combination of these structures (Table I).

Primary malignant tumors comprised the main group, encompassing 20 cases $(39 \%)$; in addition, there were 4 $(8 \%)$ desmoid tumors, $2(4 \%)$ tumors infiltrating the sternum from adjacent organs, $19(37 \%)$ recurrences or metastases from breast cancer, and $7(14 \%)$ metastases from other tumors. Twenty-one patients were male (mean age 48 years, range 16 to 75 years) and 31 were female (mean age 53 years, range 23 to 77 years).

Of the 20 primary malignant tumors (Table II), 19 were sarcomas (the main types being chondrosarcoma in 7 and osteosarcoma in 3) and 1 was a non-Hodgkin's lymphoma of the sternum. Two patients with primary malignant tumors had radiation-induced sarcomas of the sternum occurring 13 and 14 years after high-dose mediastinal irradiation (76 and 126 Gy, respectively) for Hodgkin disease. Fig. 1 illustrates 2 of the cases of primary sarcoma of the sternum.

Four patients with desmoid tumors were included in this series because their surgical management was similar to that of patients with malignant tumors; however, they were analyzed separately in the survival curves. One patient had lung cancer involving the anterior ribs and costochondral cartilages, and another had laryngeal cancer involving the upper part of manubrium.

Nineteen patients had locoregional recurrence of breast
Table II. Primary tumors

$\begin{array}{llll}\text { Malignant } & & 20 \\ \text { Sarcoma } & 7 & & \\ \text { Chondrosarcoma } & 3 & & \\ \text { Osteosarcoma } & 2 & & \\ \text { Askin's tumor (PNET) } & 1 & & \\ \text { Liposarcoma } & 1 & & \\ \text { Leiomyosarcoma } & 1 & & \\ \text { Rhabdomyosarcoma } & 1 & & \\ \text { Angiosarcoma } & 3 & & \\ \text { Soft tissue sarcoma (N.O.S.) } & & 1 & \\ \text { NH lymphoma } & & & 4 \\ \text { Desmoid } & \end{array}$

PNET, Peripheral neuroepithelioma; N.O.S., not otherwise specified; $N H$, non-Hodgkin's.

cancer: relapse was in the soft tissues of the parasternal area or at the level of the internal thoracic lymph nodes in 14 patients, and a solitary metastasis within the sternum occurred in 5 patients. Seven patients underwent surgical treatment for sternal metastases of various tumors, including 2 synovial sarcomas, 2 thyroid cancers, 2 lung cancers, and 1 gastric cancer. Thirty-nine (75\%) patients had chest pain. Seventeen patients had received preoperative radiotherapy and 12 had had preoperative chemotherapy for the chest wall lesion.

The diagnosis and surgical management were based on the history of the disease, clinical findings of a mass or pain (or both), and computed tomographic or magnetic resonance imaging (or both). In a few cases in which clinical and radiologic findings clearly indicated the presence of a primary malignant tumor of the sternum, we have performed radical en bloc resection without prior biopsy. Possible invasion of lung, pericardium, diaphragm, or clavicle was assessed by computed tomography or magnetic resonance imaging.

Follow-up was updated in July 1995, after a minimum observation of 18 months. The actuarial survival from the time of sternal resection was calculated with life table analysis according to the method of Kaplan and Meier, and statistical analysis was performed by the log-rank test.

\section{Results}

Resection and reconstruction. Complete resection of the sternum was performed in 5 patients $(10 \%)$, subtotal resection encompassing more than $50 \%$ of the sternum in 19 patients $(37 \%)$, and partial resection including less than $50 \%$ of the sternum in 28 patients $(54 \%)$ (Table III).

En bloc resection including the anterior segment of ribs was carried out in 37 patients (71\%); more than five ribs were resected in 2 patients, three or four in 18, and one or two in 17 patients; resection was limited to the costal cartilages in the remaining patients (Table III). The average defect created by resection of the sternum (and ribs) was $10 \times 7.5 \mathrm{~cm}$, and the largest defect was $27 \times 12 \mathrm{~cm}$. Concurrent 

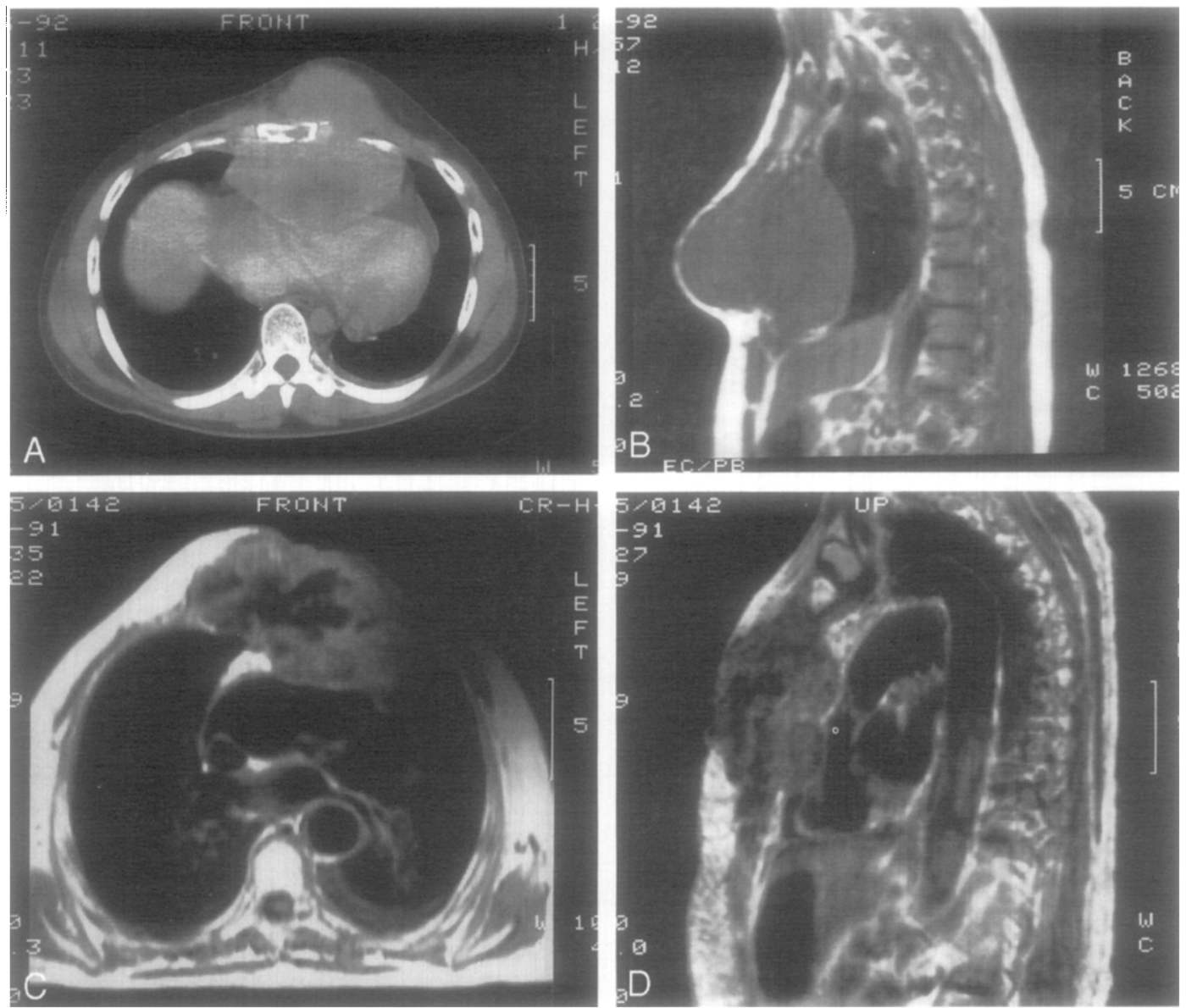

Fig. 1. Axial and sagittal views of two primary tumors of the sternum: Askin-Rosai (A and $\mathbf{B}$ ) and osteosarcoma (C and $\mathbf{D})$.

Table III. Extent of resection

\begin{tabular}{lrrrrrr}
\hline & & & & \multicolumn{2}{c}{ Total } \\
\cline { 5 - 7 } & Primary & Breast & Metastasis & No. & $\%$ \\
\hline Sternum & & & & & & \\
$\quad$ Total & 5 & 0 & 0 & 5 & 9 \\
$\quad$ Subtotal $(>50 \%)$ & 10 & 7 & 2 & 19 & 37 \\
$\quad$ Partial $(<50 \%)$ & 11 & 12 & 5 & 28 & 54 \\
Ribs & & & & & \\
0 & 8 & 5 & 2 & 15 & 29 \\
1-2 & 5 & 7 & 5 & 17 & 33 \\
$>3$ & 13 & 7 & 0 & 20 & 38 \\
Associated resections & & & & & \\
$\quad$ Lung & 5 & 6 & 1 & 12 & 23 \\
$\quad$ Pericardium & 6 & 1 & 0 & 7 & 13 \\
$\quad$ Clavicle & 6 & 2 & 3 & 11 & 21 \\
$\quad$ Diaphragm & 2 & 0 & 0 & 2 & 4 \\
\hline
\end{tabular}

resection of the lung was done in 9 patients, clavicle in 11 patients, pericardium in 3 , diaphragm in 1 , clavicle, lung, and pericardium in 3 , and diaphragm and pericardium in 1 (Table III).
Table IV. Modality of reconstruction

\begin{tabular}{lrrrrrr}
\hline & & \multicolumn{4}{c}{ Total } \\
\cline { 4 - 7 } & Primary & Breast & Metastasis & No. $\%$ \\
\hline Bone & & & & & & \\
$\quad$ Marlex mesh & 11 & 14 & 4 & 30 & 59 \\
$\quad$ Marlex composite & 7 & 0 & 1 & 8 & 11 \\
$\quad$ Other materials & 5 & 2 & 0 & 7 & 7 \\
$\quad$ None & 2 & 3 & 2 & 7 & 12 \\
Soft tissues & & & & & \\
$\quad$ Latissimus dorsi muscle & 7 & 3 & 0 & 10 & 20 \\
$\quad$ Major pectoralis muscle & 9 & 4 & 3 & 6 & 29 \\
$\quad$ Rectus abdominis muscle & 1 & 3 & 1 & 5 & 9 \\
Skin flaps & 9 & 9 & 3 & 21 & 34 \\
\hline
\end{tabular}

*Six acrylic and two silicone.

Various types of reconstruction were applied (Table IV), depending on the individual surgeon's choice: Marlex mesh was the prosthetic material used most often (38 patients, $73 \%$ ); myocutaneous flaps were used in 31 patients $(60 \%)$; in 26 patients 


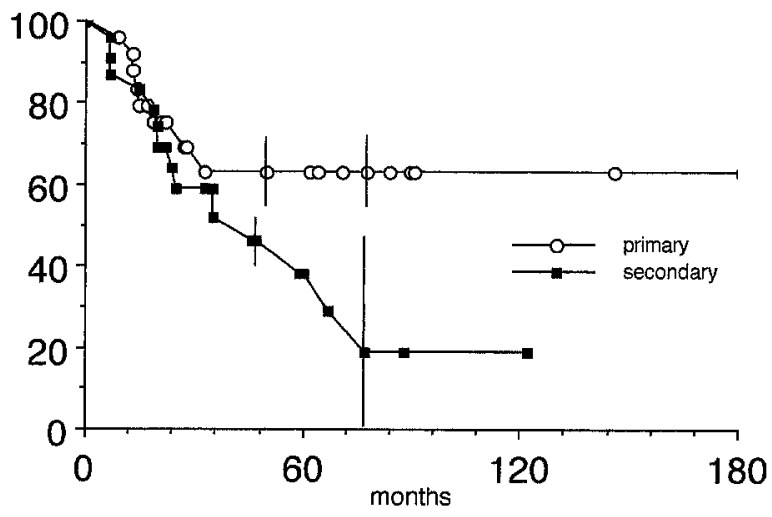

Fig. 2. Actuarial survival after radical resection according to the type of sternal involvement: primary tumor $(n=$ $24)$ versus secondary invasion (direct extension or metastasis, $n=23$ ).

prosthetic materials were used in conjunction with myocutaneous flaps $(50 \%)$; and in 5 patients flaps were used alone $(10 \%)$.

The intent of the operation was curative in 47 patients $(90 \%)$, and in 5 it was palliative because of diseased surgical margins ( 3 patients) or concurrent presence of distant metastases in other organs ( 2 patients). In such patients we decided to operate to obtain local control of the mass, reduce the thoracic pain, and improve the quality of life. All 5 patients were in good general condition and were not suitable for palliative radiotherapy for various reasons.

Postoperative course. No perioperative deaths occurred. Fourteen patients $(27 \% \pm 6 \%)$ were affected by minimal morbidity. Local complications occurred in 8 patients $(15 \%)$. In 3 patients $(1$ of whom had diabetes) the wound was necrotic: this necrosis was limited and healed spontaneously in 1 patient, but in the other 2 patients excision was necessary and a myocutaneous flap was used (in the first patient a major pectoralis flap and in the second one a latissimus dorsi flap, after the previous rectus abdominis flap had been removed). One patient had a superficial infection and four had a seroma treated with medical therapy. One patient had a local sepsis that necessitated removal of the silicone prosthesis and repeated local irrigation. It was not necessary, however, to remove the Marlex mesh. Systemic morbidity occurred in 8 patients and consisted of significant pyrexia necessitating treatment with antibiotics (in 1 patient, bilateral pneumonia).

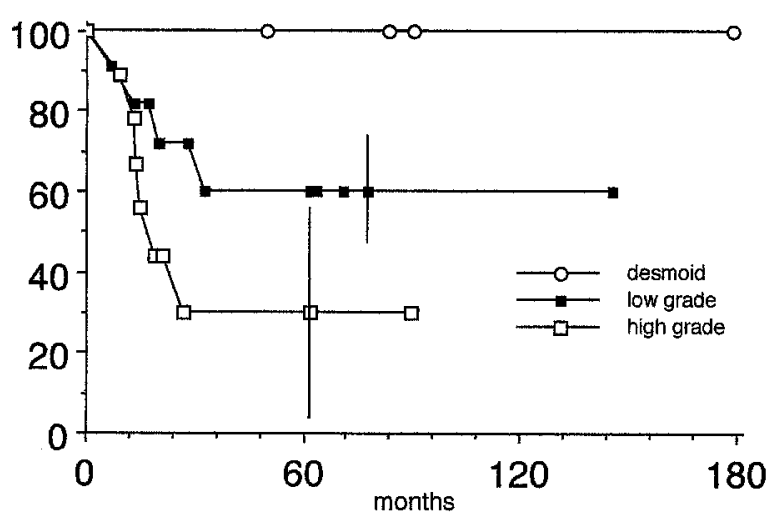

Fig. 3. Survival after radical resection for primary tumor, according to the histologic type: desmoid $(n=4)$, lowgrade sarcomas $(n=11)$, and high-grade sarcomas $(n=$ 9).

On average, the chest drains were removed 9 days after the operation (range 2 to 28 days), and after 7 days in uncomplicated cases. Average postoperative hospital stay was 14 days (range 3 to 60 days); if complicated cases are excluded, the average stay was 9 days. For prolonged loss of proteins many patients required plasma or albumin (or both); 21 patients had blood transfusion, on average 3 units per patient (range 1 to 7 units). Electrolyte alterations were infrequent and never severe.

Only 12 patients received postoperative adjuvant treatment consisting of chemotherapy (5 patients) or radiotherapy (7 patients).

Survival. After a median follow-up of 39 months, the overall 3 -year survival was $58 \%$ and the 5 -year survival $46 \%$, with a median value of 50 months and a death rate per person-year (DR) of 0.14. Among the patients who underwent radical operations, 27 $(53 \%)$ were alive (median survival 67 months) and $24(47 \%)$ were free of disease $(D R=0.12)$. None of the five patients undergoing palliative resection survived 5 years, and the median survival was only 11 months $(\mathrm{DR}=0.57)$.

Fig. 2 illustrates the actuarial survival after radical resection according to the type of sternal involvement. In 24 cases of primary tumor the 5-year survival was 63\% (median not reached) and in 23 cases of secondary invasion (direct extension or metastasis) the 5-year survival was $38 \%$, with a median value of 35 months ( $\mathrm{DR}=0.07$ and 0.18 , respectively).

Fig. 3 illustrates the survival of patients with primary tumors according to the histologic diagno- 
sis. All 4 patients with desmoid tumors were alive and free of disease at 5 years, whereas the 5-year survival was $60 \%$ for the 11 patients with low-grade sarcoma and 30\% (median 17 months) for the 9 patients with high-grade sarcoma (DR $=0.08$ and 0.27 , respectively).

In patients who underwent sternal resection for recurrent breast cancer (Fig. 4), the 5-year survival was similar regardless of whether the patient had direct extension to the chest wall or distant bone metastasis $(48 \%$ vs $60 \%$; DR $=0.15$ and 0.13 , respectively), with a remarkably long median survival. However, none of the 5 patients with metastatic tumors other than breast cancer survived, and the median survival was only 12 months (not shown).

Regarding symptomatic benefit, none of the patients operated on for advanced breast cancer had local tumor relapse or recurrence of symptoms, namely chest pain, during the follow-up period.

\section{Discussion}

Resection of large portions of the chest wall has been a complex problem in the past on account of technical intraoperative difficulties, surgical complications, and respiratory failure caused by the chest wall instability and paradoxic respiratory movements. Present-day cooperation between thoracic and plastic surgeons allows more aggressive resections, ${ }^{2}$ and neither the size nor the position of the chest wall defect limits surgical management, because resection and reconstruction are performed in a single operation that provides immediate chest wall stability.

Surgery is the treatment of choice for patients with primary tumors of the chest wall including the sternum. ${ }^{1,10}$ For some authors, survival is not significantly different between primary and recurrent sarcomas. $^{4}$

For primary malignant tumors of the chest wall, a 5 -year survival range of $46 \%{ }^{5}$ to $66 \%{ }^{11}$ has been reported, with a wide difference between high-grade and low-grade sarcomas. Five-year survival ranges from $16.6 \%$ for peripheral neuroepitheliomas to $89 \%$ for chondrosarcomas. ${ }^{12}$ Others report that 5 -year survival was $14 \%$ in osteosarcomas and $80 \%$ in chondrosarcomas. ${ }^{6}$

After a median follow-up of 31.5 months, the survival was $79 \%$ for primary tumors and $33 \%$ for metastatic tumors ${ }^{2}$; in another series, survival was $59 \%$ without any difference among primary and recurrent tumors. ${ }^{4}$ Our series confirms that low-

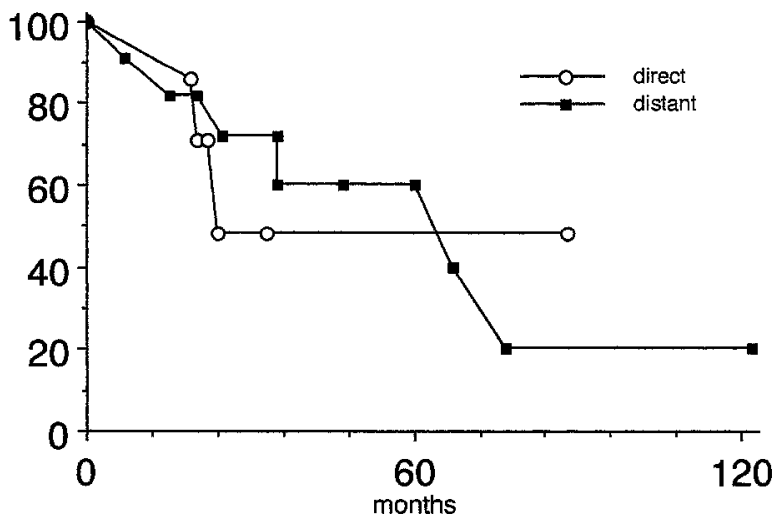

Fig. 4. Survival after radical resection for recurrent breast cancer: direct chest wall extension $(n=7)$ versus distant bone metastasis $(n=11)$.

grade primary sarcomas have a better prognosis than high-grade tumors ( $60 \%$ vs $30 \%$ at 5 years; see Fig. 3), although because of the limited number of observations the difference does not reach statistical significance.

Desmoid tumors are considered to be a benign fibromatosis or a low-grade fibrosarcoma by pathologists, but they should be resected with wide margins in normal tissue, as are primary malignant tumors, to avoid recurrence. ${ }^{2}$

Sarcomas arising in the sternum after extensive mediastinal irradiation for lymphomas may be difficult to treat. In fact, the limits between the tumor and radiation-induced fibrosis may not be properly defined, and the tumor is likely to recur despite undiseased resection margins at pathologic examination. In this series, the 2 patients with sarcomas of the sternum induced by mediastinal irradiation for Hodgkin's disease had widespread mediastinal recurrence a few months after radical sternectomy and died within 2 years of the operation.

On the other hand, the role of surgery remains a subject of controversy for recurrence and metastasis of breast cancer involving the chest wall and sternum, ${ }^{8,13}$ in which surgery may be considered for simple palliation and local control of the disease. The actuarial 5-year survival after resection for breast cancer recurrences ranges from $34 \%^{14}$ to $63 \%{ }^{15}$ in the literature. A few authors have advocated a radical surgical approach for patients with solitary breast cancer recurrence or metastasis involving the chest wall and sternum, because survival may be improved. ${ }^{7,9}$ It has been suggested that sternal metastases may remain solitary for a long 
time and that their evolution may be different from that of vertebral metastases, possibly because of the lack of communication with paravertebral venous plexus through which cancer cells spread to other bones. ${ }^{8}$ In our opinion, surgical therapy should be considered occasionally for isolated bone metastases of breast cancer as part of a combined multimodality approach that includes radiotherapy, chemotherapy, and hormone therapy. Adjuvant chemotherapy and hormone therapy increase the disease-free survival and overall survival. ${ }^{16}$

The prognosis of local chest wall recurrence or metastasis of breast cancer is better in the absence of internal thoracic and mediastinal lymph node metastases; if mediastinal lymph nodes are involved, the chance of progression with metastatic dissemination is much higher. ${ }^{8}$ Surgery may still be considered in such cases, whenever adequate local control cannot be achieved by radiotherapy or chemotherapy, to palliate thoracic pain, inflammation, infections, and bleeding associated with the recurrence. In the present series, the 5-year survival was equally good in patients with direct extension to the chest wall or distant bone metastasis (48\% vs $60 \%$, see Fig. 4).

Regarding the margin of resection, most authors recommend that the incision be placed 2 to $4 \mathrm{~cm}$ from the margin of the tumor or the irradiated tissue. $^{7}$ Others ${ }^{2}$ advocate that a minimum of $4 \mathrm{~cm}$ of normal tissue be removed en bloc with fullthickness resection of the involved bone (manubrium, sternal body, or whole sternum) and corresponding anterior tract of the ribs bilaterally, one uninvolved rib and the intercostal space above and below. ${ }^{4}$

Many materials had been used in the past for reconstruction of anterior defects of the chest wall: fascia lata,${ }^{17}$ rib grafts, ${ }^{18}$ large cutaneous flaps, and contralateral breast. ${ }^{19}$ A great advance was made when the use of a latissimus dorsi flap with preservation of the vascular thoracodorsal system was introduced. ${ }^{3,20}$ The strategy for reconstruction now has to be based on the extent of skeletal and soft tissue defect. The potential combinations of prosthetic materials and myocutaneous flaps are countless, and often more than one option can effectively deal with the individual patient's problem. The need for skeletal reconstruction depends on the size and site of resection ${ }^{2}$ : reconstruction is necessary for the sternum and for the anterior and lateral tracts of the ribs, whereas posterior defects may not require reconstruction if they are fully covered by the scap- ula without any risk of inward rotation ${ }^{5}$ or if they can be stabilized by adjacent muscles. For some authors sternal and posterior defects require less stabilization than anterior and lateral defects. ${ }^{2,21}$ Various options of prosthetic materials are possible. Marlex mesh, polytetrafluoroethylene patch, ${ }^{*}$ and Prolene mesh (Ethicon, Inc., Somerville, N.J.) are well tolerated, easy to handle, and must be sutured under tension, which improves the stability of the chest wall. ${ }^{3,4}$ Polytetrafluoroethylene has the advantage of being impervious to the flow of air and fluids. ${ }^{22}$ Neither Marlex, Vicryl (Ethicon), nor Prolene mesh has this characteristic, but in our experience a transient flow of serum or blood through the prosthesis did not create any problem. Among the various prosthetic meshes with similar physical properties, Marlex mesh remains the most widely used because of its solidity, manageability, and long-term tolerability, the virtual absence of foreign body reactions or septic complications, and the low cost. After total sternectomy or large resections involving the lateral aspect of the chest wall and more than four ribs, the Marlex mesh-methacrylate composite offers the best results in terms of fixation, protection of endothoracic organs, and lung expansion. ${ }^{6,23}$ The mesh-methacrylate composite is manufactured at the time of the operation, when the size and shape of the prosthesis can be determined, and provides excellent functional and cosmetic results. Paradoxic movement of the chest can be prevented with this prosthesis, even with large defects. Nonetheless, in our experience, when a partial sternal resection is performed with preservation of the anterior chest wall continuity (manubrium or a part of the sternal body with adjacent ribs), it is possible to use the simple Marlex mesh fixed under tension to obtain adequate skeletal stability.

For the reconstruction of soft tissue defects, myocutaneous flaps have replaced other tissues, such as simple skin flaps or breast transposition, because of their safety and long-term reliability. This aspect is particularly critical in managing breast cancer recurrence because of the damage caused by the previous operation and radiation to the surrounding tissues. Depending on the specific circumstances, different myocutaneous flaps can be used, alone or in combination: major pectoralis muscle to repair upper anterior defects, transverse rectus or serratus muscle for anterior and lateral defects, and latissimus

\footnotetext{
${ }^{*}$ Gore-Tex patch, registered trade name of W. L. Gore \& Associates, Inc., Elkton, Md.
} 
dorsi muscle for any anterior or lateral defects. The omentum has been proposed as a valid alternative to muscular flaps for its excellent blood supply ${ }^{24}$ and good performance in potentially infected areas. Clinical results are favorable, ${ }^{25}$ but the obvious disadvantages are the need for additional laparotomy and alternative sources of skin cover.

In conclusion, our results confirm that even extensive resection of the sternum for primary or secondary tumors followed by reconstruction with prosthetic material or a myocutaneous flap is a safe and effective treatment. Various combinations of myocutaneous flaps and prosthetic materials can be used, and the choice of technique depends on size, position of the defect, and surgeon's preference. It is clear, however, that major resections of the sternum, en bloc with pericardium, lung, or diaphragm, can be performed with no mortality, minimum morbidity, and reasonable duration of the hospital stay. In primary tumors, as demonstrated by a recent series, ${ }^{6}$ radical resection may provide a permanent eradication of the disease and a good prospect for longterm survival. Moreover, in patients with recurrent breast cancer, sternal resection offers not only a significant and permanent palliation but also a prolonged survival.

\section{REFERENCES}

1. McCormack PM, Bains MS, Beattie ED Jr, et al. New trends in skeletal reconstruction after resection of chest wall tumors. Ann Thorac Surg 1981;31:45-56.

2. Pairolero PC, Arnold PG. Chest wall tumors: experience with 100 consecutive patients. J Thorac Cardiovasc Surg 1985;90: 367-72.

3. Morgan RF, Edgerton MT, Wanebo HJ, Daniel TM, Spotnitz WD, Kron IL. Reconstruction of full thickness chest wall defects. Ann Surg 1988;207:707-16.

4. Perry RR, Venzon D, Roth JA, Pass HI. Survival after surgical resection for high-grade chest wall sarcomas. Ann Thorac Surg 1990;49:363-9.

5. Eng J, Sabanathan S, Mearns AJ. Chest wall reconstruction after resection of primary malignant chest wall tumors. Eur J Cardiothorac Surg 1990;4:101-4.

6. Martini N, Huvos AG, Burt ME, et al. Predictors of survival in malignant tumors of the sternum. $\mathrm{J}$ Thorac Cardiovasc Surg 1996;111:96-106.

7. McCormack PM, Bains MS, Burr ME, Martini N, Chaglassian T, Hidalgo DA. Local recurrent mammary carcinoma failing multimodality therapy: a solution. Arch Surg 1989; 124:158-61.
8. Noguchi S, Miyauchi K, Nishizawa Y, Imaoka S, Koyama H, Iwanaga $T$. Results of surgical treatment for sternal metastasis of breast cancer. Cancer 1988;62:1397-401.

9. Brower ST, Weinberg H, Tartter PI, Camunes J. Chest wall resection for locally recurrent breast cancer: indications, technique and results. J Surg Oncol 1992;49:189-95.

10. Kroll SS, Schusterman MA, Larson DL, et al. Long term survival after chest wall reconstruction with musculocutaneous flaps. Plast Reconstr Surg 1990;86:697-701.

11. Gordon MS, Hajdu SI, Bains MS, Burr ME. Soft tissue sarcomas of the chest wall. J Thorac Cardiovasc Surg 1991; 101:843-54.

12. Graeber GM, Snyder RJ, Fleming AW, et al. Initial and long-term results in the management of primary chest wall neoplasms. Ann Thorac Surg 1982;34:664-73.

13. Hathaway CL, Rand RP, Moe R, Marchioro T. Salvage surgery for locally advanced and locally recurrent breast cancer. Arch Surg 1994;129:582-7.

14. Muscolino G, Valente M, Lequaglie C, Ravasi G. Correlation between first disease-free interval from mastectomy to second disease-free interval from chest wall resection. Eur J Surg Oncol 1992;18:49-52.

15. Stotter A, Kroll S, McNeese M, Holmes F, Oswald MJ, Romsahl M. Salvage treatment for loco-regional recurrence following breast conservation therapy for early breast cancer. Eur J Surg Oncol 1991;17:231-6.

16. Probsfeld MR, O'Connell TX. Treatment of locally recurrent breast carcinoma. Arch Surg 1989;124:1127-30.

17. Watson WS, James AG. Fascia lata grafts for chest wall defects. J Thorac Surg 1947;16:399-406.

18. Bisgard JD, Swenson SA. Tumors of the sternum: report of a case with special operative technic. Arch Surg 1948;56:570-7.

19. Maier HC. Surgical management of large defects of the thoracic wall. Surgery 1947;22:169-78.

20. Campbell DA. Reconstruction of the anterior thoracic wall. J Thorac Surg 1950;19:456-61.

21. Hidalgo DA, Saldana EF, Rusch VW. Free flap chest wall reconstruction for recurrent breast cancer and radiation ulcers. Ann Plast Surg 1993;30:375-80.

22. Hyans P, Moore JH, Sinha L. Reconstruction of the chest wall with e-PTFE following major resection. Ann Plast Surg 1992;29:321-7.

23. Chapelier A, Macchiarini P, Rietjens M, Lenot B, Margulis A, Petit JY, et al. Chest wall reconstruction following resection of large primary malignant tumors. Eur J Cardiothorac Surg 1994;8:351-7.

24. Jurkiewicz MJ, Arnold PG. The omentum: an account of its use in the reconstruction of the chest wall. Ann Surg 1977; 185:548-54.

25. Al-Kattan KM, Breach NM, Kapplan DK, Goldstraw P. Soft-tissue reconstruction in thoracic surgery. Ann Thorac Surg 1995;60:1372-5. 